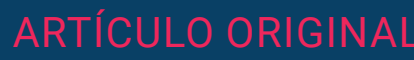

\title{
Impacto de un programa de prevención de infecciones en la conducta de cirujanos y anestesistas en un hospital público
}

\author{
Recibido: 19/9/19 Aceptado: 28/9/20
}

RESUMEN

Introducción: El control de las infecciones de sitio quirúrgico (ISQ) es importante en los programas de vigilancia y control de infecciones nosocomiales. Hay evidencias de que hasta un $60 \%$ podrían evitarse. Nuestro objetivo fue determinar si el porcentaje de cumplimiento de las medidas de prevención de ISQ fue mayor luego de la implementación de un programa en un hospital público.

Material y métodos: Estudio comparativo inter-sujeto, observacional, retrospectivo y transversal. Se analizaron fichas de registro de cumplimiento de medidas de prevención pre y post instrumentación del programa, que consistió en entrega de ropa quirúrgica al ingreso al quirófano, difusión de las recomendaciones de prevención de ISQ y auditoría diaria. Variables: categoría profesional, uso adecuado de barbijo, de ambo y de camisolín, higiene de manos quirúrgica, cambio de ropa cuando se mancha, y manejo de efectos personales dentro del quirófano. Los datos fueron analizados empleando VCCstat3.0. Se estimaron intervalos de confianza del $95 \%$.

Resultados: En el control de la categoría "Profesional" hubo diferencias significativas en todas las variables auditadas entre cirujanos y anestesistas. Por lo tanto, se realizó un análisis de las poblaciones por separado. Cirujanos y anestesistas mejoraron el cumplimiento de ambo adecuado, cambio de ropa cuando se mancha y manejo de efectos personales dentro del quirófano. Los anestesistas, además,
Elena Rivera ${ }^{1}$, Martín Hojman² y Marcela DiPaolo³

'Departamento de Enfermería del Hospital General de
Agudos “Bernardino Rivadavia", Ciudad Autónoma de
Buenos Aires, Argentina.
² Departamento de Medicina del Hospital General de
Agudos “Bernardino Rivadavia", Ciudad Autónoma de
Buenos Aires, Argentina.
${ }^{3}$ Departamento Quirúrgico del Hospital General de Agudos
"Bernardino Rivadavia”, Ciudad Autónoma de Buenos Aires,
Argentina.

Dirección para la correspondencia: Lic. Elena Rivera. Av. Las Heras 2670, Infectología y Control de Infecciones, CABA. Tel 4809-2000.

Email: riverarelena@gmail.com

Se deja constancia que los contenidos del material a ser evaluado no han sido publicados anteriormente. Ninguno de los autores declara presentar conflicto de intereses en relación a ésta publicación.

presentaron mejoras significativas en el uso adecuado de barbijo e higiene de manos quirúrgica.

Conclusiones: La instrumentación del programa fue exitosa. Es importante continuar con los esfuerzos de la mejora. El efecto en las tasas de ISQ es un dato a medir.

Palabras clave: medidas de prevención, infecciones quirúrgicas, comportamiento en quirófano, anestesistas, cirujanos. 


\section{Introducción}

Las infecciones asociadas al cuidado de la salud (IACS) se definen como aquellas infecciones, sin evidencia de que estuvieran presentes o incubándose en el periodo de admisión a los cuidados sanitarios, cuyo origen más probable es la actividad asistencial y que resultan de una reacción adversa a la presencia de un agente infeccioso o de su toxina (1). Las infecciones de sitio quirúrgico (ISQ) son un tipo de IACS que ocurre después de una intervención quirúrgica en una zona del cuerpo donde se llevó a cabo la operación, implicando a la piel, a los tejidos y órganos o material implantado, revelándose como una combinación de signos y síntomas que muestran la infección (2).

Las ISQ ocupan un lugar destacado en los programas de vigilancia y control de la infección nosocomial (3), ya que ostentan todas las características para convertirse en prioritarias para su prevención: elevada prevalencia (4), demostrada gravedad (5), gran incremento de costos sanitarios directos e indirectos (6) y disponibilidad de medidas de prevención efectivas $(7,8)$ científicamente probadas para cada tipo de procedimiento quirúrgico (9).

Existen variadas evidencias de que hasta un $60 \%$ de las ISQ serían evitadas si se aplicaran adecuados programas de prevención $(6,8,10)$-basados en el conocimiento de diversos factores de riesgo que predisponen al paciente para desarrollar infección y en un entendimiento de la microbiología de la ISQ - y verificando su cumplimiento (11), ya que los paquetes de medidas o "bundles" han demostrado una reducción de tasas de ISQ $(12,13)$, aunque sus resultados pueden variar según diversos factores, entre ellos la elección de las medidas individuales que los constituyen.

El primer reservorio de microorganismos que causan ISQ es la flora endógena del propio paciente. Muchas heridas pueden verse afectadas a partir de la misma, por lo cual ha cobrado vital importancia la preparación pre quirúrgica con el fin de disminuir la carga microbiológica.

Las fuentes exógenas incluyen al medio ambiente del quirófano y al personal que se desempeña en su interior. La fuente primaria de bacterias aerobias en el quirófano la constituye el equipo quirúrgico. El personal de salud dispersa los microorganismos, especialmente Staphylococcus a partir de su propia piel. Por esta razón, las medidas de control de infecciones deben estar dirigidas a minimizar esta posibilidad. Algunas de ellas son higiene de manos quirúrgica y vestimenta quirúrgica.
La expresión "vestimenta quirúrgica" designa la indumentaria usada por el equipo quirúrgico: ambo (chaqueta y pantalón), gorro, barbijo, camisolín, guantes y cobertores de calzado y/o zuecos.

Datos experimentales han mostrado que los microorganismos pueden desprenderse del cabello, la piel o las membranas mucosas del personal de quirófano. Sin embargo, son muy pocos los estudios clínicos controlados que han evaluado la relación existente entre el uso de vestimenta quirúrgica y el riesgo de ISQ. No obstante, el uso de barreras es una medida prudente para minimizar la exposición de los pacientes a estos y a su vez protege al personal de salud de los patógenos de los pacientes.

Según las normas, toda persona que ingrese al área quirúrgica deberá colocarse un ambo (pantalón y camisa) que debe cubrir la mayor superficie corporal que fuera posible (16). La utilización de ambos adecuados mostró ser un factor importante en la prevención de estas infecciones (15).

Cuando la vestimenta quirúrgica queda visiblemente sucia o manchada, debe ser reemplazada por otra; cuando se humedece favorece el pasaje de microorganismos provenientes de la piel de los cirujanos hacia el campo operatorio.

Los datos que demuestran el posible efecto protector del uso de barbijo quirúrgico en relación con el riesgo de ISQ son limitados. Sin embargo, existe una fundada presunción que indica la necesidad de usar barbijo quirúrgico siempre, durante todas las operaciones y mientras se permanezca en el quirófano $(19,20)$.

El objetivo de la higiene de manos quirúrgica es disminuir los patógenos residentes y transitorios que están en las manos, las uñas y los antebrazos sobre la piel intacta, utilizando un antiséptico no irritante, de amplio espectro microbicida, de acción rápida y con un efecto prolongado $(17,18)$.

\section{Justificación}

En un relevamiento reciente de uso de antibióticos en la institución se evidenció que las áreas de internación quirúrgicas presentan un alto consumo de antimicrobianos comparado con el de otras áreas (14). En recorridas semanales del equipo de Control de Infecciones se observaron desvíos en el cumplimiento de las normas de prevención mayoritariamente por parte de anestesistas y cirujanos, por lo que se consideró oportuno realizar una investigación 
para evaluar el cumplimiento de algunas de las medidas de prevención de ISQ por parte ellos, evaluando la adherencia a las medidas de prevención.

Una medida de prevención es definida como una acción o acciones intencionalmente realizadas por el equipo de salud, con el objetivo de reducir el riesgo de ISQ.

El objetivo de nuestro trabajo fue determinar si el porcentaje de cumplimiento de las medidas de prevención de infecciones quirúrgicas es mayor luego de la implementación de un programa de prevención de ISQ en un hospital público.

\section{Materiales y métodos}

Estudio comparativo inter-sujeto, observacional, retrospectivo (junio 2017 a febrero 2018) y transversal. Se realizaron observaciones sobre la conducta del personal quirúrgico (cirujanosy anestesistas) en los quirófanos del Hospital General de Agudos "Bernardino Rivadavia" y el resultado fue plasmado en fichas de registro. De esas fichas fueron tomados los datos. Fueron excluidas las fichas en las que los cirujanos o anestesistas presentaron, al momento de la recolección datos, signos o síntomas de enfermedades transmisibles (exantemas, anginas, conjuntivitis, patologías dérmicas supurativas) que impidieran su participación en el acto quirúrgico o de patologías alérgicas que impidieran la correcta realización de la técnica de higiene de manos quirúrgica. Se registraron los siguientes datos: categoría profesional, barbijo adecuado, ambo adecuado, camisolín adecuado, higiene de manos quirúrgica, cambio de ropa cuando se mancha, manejo de objetos personales dentro del quirófano (colocar la mochila o el bolso dentro de bolsa plástica).

Se seleccionaron cinco instrumentadoras del turno mañana para que realizaran las observaciones pre y post instrumentación del programa de prevención de ISQ. Las licenciadas fueron capacitadas por los autores.

La medición basal de cumplimiento de las medidas de prevención de infecciones quirúrgicas se realizó en junio-julio de 2017; luego se efectuó la devolución de los datos al jefe del Departamento de Cirugía, jefa de Anestesia y a la jefa de instrumentadoras.

Se implementó el siguiente programa de prevención de ISQ:

- Entrega de la ropa quirúrgica adecuada al ingreso al quirófano.
- Difusión de las recomendaciones de prevención de infección de sitio quirúrgico, elaboradas por Infectología y Control de Infecciones, por parte de la jefa de Anestesia y el jefe del Departamento de Cirugía.

- Auditoría diaria por parte de las instrumentadoras.

La segunda medición de cumplimiento de las medidas de prevención de infecciones quirúrgicas se realizó en enerofebrero de 2018.

\section{Variable dependiente: medidas de prevención de ISQ:}

- Barbijo adecuado. Utilizar un barbijo nuevo por cirugía. El mismo debe cubrir la nariz y la boca, y ser utilizado durante toda la cirugía.

- Ambo adecuado: ambo de tela, limpio, provisto por el hospital y colocado dentro del quirófano.

- Camisolín adecuado. Camisolín estéril de tela o descartable.

- Higiene de manos quirúrgica:

a. Debe ser realizada antes de cada cirugía.

b. Con jabón antiséptico.

c. Durante por lo menos dos minutos.

d. Utilizando cepillo solo para las uñas.

e. Las manos, antebrazos y codos se deben friccionar con esponjas que no lesionen la piel.

f. Las manos serán secadas con compresas estériles.

* Opción para el lavado de manos quirúrgico: lavado con solución jabonosa seguido de frotado de manos con solución de base alcohólica.

- Cambio de ropa cuando se mancha. El ambo se debe cambiar cuando esté manchado con sangre o cualquier otro fluido potencialmente infectante.

Manejo de efectos personales dentro del quirófano (colocar la mochila o bolso dentro de una bolsa plástica). Debido a la falta de armarios para que los profesionales 
guarden sus pertenencias se recomienda colocarlos dentro de bolsa plástica.

\section{Variable independiente:}

- Periodos: se categorizó en 2017 (junio-julio) y 2018 (enero y febrero).

\section{Variable a controlar:}

- Categoría profesional. Rama de la ciencia de la salud a la que se dedica, dividida en: anestesistas (médicos especializados en anestesia, de planta y residentes) y cirujanos (médicos especializados en cirugía, de planta y residentes).

\section{Lugar:}

Hospital Bernardino Rivadavia.

Los datos fueron volcados en una base de datos (Microsoft Excel 97) y luego analizados empleando el paquete estadístico (SPSS 11.5 y VCCstat 3.0). Para todas las variables se estableció su distribución de frecuencias y/o porcentajes en relación con el total de casos. Para aquellas medidas en escala ordinal o superior se computaron los siguientes datos estadísticos: número de casos, valor mínimo hallado, valor máximo hallado, media aritmética, desvío típico y error típico. Cuando fue necesario se estimaron los intervalos de confianza del 95\% (IC95) y se realizaron pruebas de significación (Test Chi cuadrado). El nivel de significación se estableció en alfa 0.05 .

\section{Reparos éticos}

Antes de dar inicio esta investigación se presentó el protocolo de investigación científica al Comité de Ética en Investigación (CEI) del Hospital B. Rivadavia para su valoración y aprobación (datos del CEl Hospital Rivadavia disponible en la página web oficial www.comiterivadavia.com.ar).

Esta investigación respetóy cumplió con todas las legislaciones y reglamentaciones pertinentes para este estudio a las cuales adhiere el CEI según su Manual de Procedimientos operativos (POE) vigentes al momento de la ejecución de esta investigación.

Se respetó la confidencialidad de la información personal incluida en fichas de registro, garantizando la no utilización de la información obtenida en perjuicio de los sujetos en estudio.
Los investigadores implementaron métodos que permiten la codificación/anonimización de los datos de los sujetos con el objeto de resguardar la información surgida a partir de la recolección, uso y transferencia de datos, según los términos de la Ley 1845 (Ley de protección de los datos personales vigente en la Ciudad de Buenos Aires).

Los investigadores presentaron los informes de inicio de estudio (primer caso incluido) y de avance con la frecuencia que fue establecida por el CEl e informe de finalización de la investigación.

\section{Confidencialidad de los datos}

Para proteger la confidencialidad de los datos los mismos se mantuvieron en gabinetes de archivos cerrados ubicados en Infectología y Control de Infecciones, a los cuales únicamente tuvo acceso el personal autorizado del estudio. La información que se incluyó en la base de datos no permitió la identificación personal de los incluidos debido a que se aplicó el procedimiento de codificación/anonimización.

\section{Resultados}

Se analizaron los datos de 129 fichas de registro de cumplimiento de medidas de prevención de ISQ de Infectología y Control de Infecciones. Cada ficha incluyó a dos cirujanos y a dos anestesistas.

Como se planteó que había variables que podían influenciar los resultados, se controló la variable "categoría profesional" para determinar si el comportamiento de los cirujanos era diferente al de los anestesistas en relación a las medidas de prevención quirúrgicas, con los siguientes resultados (Tabla 1):

1. Los cirujanos tuvieron en general un porcentaje de cumplimiento mayor que los anestesistas en relación a las siguientes variables: barbijo adecuado, ambo adecuado, camisolín adecuado, higiene de manos quirúrgica y cambio de ropa cuando se mancha.

2. Los anestesistas tuvieron un porcentaje de cumplimiento mayor que los cirujanos en relación a la variable: manejo de efectos personales dentro del quirófano (colocar la mochila o el bolso dentro de la bolsa plástica).

Dado que se encontró diferencia estadísticamente significativa, podemos afirmar que los cirujanos no se comportan igual que los anestesistas en relación a las medidas de prevención de ISQ. Por esa razón los resultados se presentaron por separado 


\section{Tabla 1. Control de categoría profesional en relación a las medidas de prevención de ISQ}

\begin{tabular}{|l|c|c|c|}
\hline Medidas de prevención & $\begin{array}{c}\text { Cirujanos } \\
(\mathrm{n}=259)\end{array}$ & $\begin{array}{c}\text { Anestesistas } \\
(\mathrm{n}=255)\end{array}$ & $\begin{array}{c}\mathbf{p} \\
\text { Uso adecuado de barbijo }\end{array}$ \\
\hline Uso adecuado de ambo & $94 \%$ & $68 \%$ & 0,0008 \\
\hline Uso adecuado de camisolín & $96 \%$ & $22 \%$ & $<0,0001$ \\
\hline Higiene de manos quirúrgica & $95 \%$ & $55 \%$ & $<0,0001$ \\
\hline Cambio de ropa cuando se mancha & $32 \%$ & $11 \%$ & $<0,0001$ \\
\hline Manejo de efectos personales dentro del quirófano & $65 \%$ & $78 \%$ & 0,0008
\end{tabular}

Fuente: Fichas de registro de cumplimiento de medidas de prevención de ISQ de Infectología y Control de Infecciones $2017-2018$

\section{Tabla 2. Comparación del porcentaje de cumplimiento de las medidas de prevención de ISQ de los} cirujanos de 2018 con respecto al reportado en 2017

\begin{tabular}{|c|c|c|c|}
\hline Medidas de prevención & $\begin{array}{l}\text { Pre programa } \\
(2017) n=119\end{array}$ & $\begin{array}{l}\text { Post programa } \\
(2018) n=255\end{array}$ & $\mathbf{p}$ \\
\hline Uso adecuado de barbijo & $93 \%$ & $95 \%$ & ns \\
\hline Uso adecuado de ambo & $38 \%$ & $88 \%$ & $<0,0001$ \\
\hline Uso adecuado de camisolín & $94 \%$ & $97 \%$ & ns \\
\hline Higiene de manos quirúrgica & $94 \%$ & $96 \%$ & ns \\
\hline Cambio de ropa cuando se mancha & $21 \%$ & $41 \%$ & 0,0005 \\
\hline Manejo de efectos personales dentro del quirófano & $53 \%$ & $75 \%$ & 0,0003 \\
\hline
\end{tabular}

Fuente: Fichas de registro de cumplimiento de medidas de prevención de ISQ de Infectología y Control de Infecciones 2017-2018

\section{Tabla 3. Comparación del porcentaje de cumplimiento de las medidas de prevención de ISQ de los anestesistas de 2018 con respecto al reportado en 2017}

\begin{tabular}{|c|c|c|c|}
\hline Medidas de prevención & $\begin{array}{l}\text { Pre programa } \\
(2017) n=115\end{array}$ & $\begin{array}{c}\text { Post programa } \\
(2018) n=140\end{array}$ & p \\
\hline Uso adecuado de barbijo & $62 \%$ & $74 \%$ & 0,0439 \\
\hline Uso adecuado de ambo & $14 \%$ & $69 \%$ & $<0,0001$ \\
\hline Uso adecuado de camisolín & $19 \%$ & $25 \%$ & ns \\
\hline Higiene de manos quirúrgica & $32 \%$ & $73 \%$ & $<0,0001$ \\
\hline Cambio de ropa cuando se mancha & $3 \%$ & $16 \%$ & 0,0008 \\
\hline Manejo de efectos personales dentro del quirófano & $70 \%$ & $86 \%$ & 0,0018 \\
\hline
\end{tabular}

Fuente: Fichas de registro de cumplimiento de medidas de prevención de ISQ de Infectología y Control de Infecciones 2017-2018

(Tablas 2 y 3$)$.

Con respecto a los cirujanos, en 2018 el porcentaje de cumplimiento de ambo adecuado, cambio de ropa cuando se mancha y manejo de efectos personales fue mayor que el reportado en 2017. No hubo diferencias entre 2017 y 2018 en el porcentaje de cumplimiento de barbijo adecuado, higiene de manos quirúrgica y camisolín adecuado. El valor del indicador se mantiene elevado.
Con respecto a los anestesistas, en 2018 el porcentaje de cumplimiento de barbijo adecuado, ambo adecuado, higiene de manos quirúrgica, cambio de ropa cuando se mancha y colocar la mochila o bolso dentro de bolsa plástica es mayor que el reportado en 2017. No hubo diferencias entre 2017 y 2018 en el porcentaje de cumplimiento de camisolín adecuado. El valor del indicador se mantiene bajo. 


\section{Discusión}

Muchas veces, el comportamiento de los efectores de salud en los actos quirúrgicos no es el adecuado. La urgencia, la ponderación del acto quirúrgico en sí por sobre lo demás, las conductas individuales y la dificultad para el cumplimiento de las normas se asocian a los problemas edilicios y de insumos que pudieran ocurrir, dificultando el control de la ocurrencia de infección de sitio quirúrgico.

Nuestro trabajo valora las consecuencias de la implementación de un programa destinado a mejorar el cumplimiento de medidas de prevención de ISQ, con la mirada puesta en los cirujanos y anestesistas.

Inicialmente, previa a la instrumentación del citado programa, nuestros resultados mostraron un cumplimiento significativamente menor de las medidas de prevención de ISQ por parte de los anestesistas en comparación con los cirujanos. Es común que algunos anestesistas no se perciban a sí mismos como personal de riesgo ya que no están en contacto directo con el campo quirúrgico, por lo que probablemente no consideren tan necesario el cumplimiento de las normas relacionadas al control de infecciones. En la medición basal, los resultados de los cirujanos tuvieron un valor promedio de cumplimiento del $65,56 \%$. Este valor superior al $50 \%$ podría deberse a que el objetivo del cirujano es curar al paciente, siendo una prioridad la prevención de ISQ.

Después de la implementación del programa los cirujanos tuvieron un aumento en el cumplimiento de la utilización de ambo adecuado, en el cambio de ropa quirúrgica cuando se mancha y en la manipulación de efectos personales dentro del quirófano (guardado de mochila o bolso dentro de bolsa plástica). Con respecto al uso de barbijo adecuado, higiene de manos quirúrgica y uso de camisolín adecuado no se evidenciaron cambios. Aunque la medición realizada previa a la implementación del programa arrojó resultados altos de cumplimiento, por lo que no se esperaban mejoras significativas.

Los anestesistas también mostraron mejoras en los mismos tópicos que los cirujanos, agregándose uso adecuado de barbijo e higiene de manos quirúrgica.

Es de remarcarse que, a pesar de las mejoras en cada grupo, se vio en los anestesistas un cumplimiento menor de las medidas auditadas, excepto en el manejo de efectos personales en quirófano (guardado de mochila o bolso en bolsa plástica).
La instrumentación del programa fue exitosa en términos de cumplimiento ya que se logró mejorar la cultura de seguridad de anestesistas y cirujanos.

Sería importante, en futuros trabajos, evaluar el impacto del programa en la tasa de infecciones quirúrgicas de la institución y la correlación de cumplimiento de normas de los cirujanos con los anestesistas.

Es importante seguir efectuando mediciones y evaluaciones periódicas como parte de un programa de mejora continua. Más trabajo, de todas maneras, es necesario, y conforma un desafío lograr una mejor llegada a los anestesistas con el fin de mejorar la adherencia a las medidas de control de infecciones dentro de quirófano. 


\section{Bibliografía}

1. T. C. Horan, M. Andrus, M. A. Dudeck - CDC/NHSN surveillance definition of health care-associated infection and criteria for specific types of infections in the acute care setting-Am J Infect Control., 36 (2008), pp. 309-332 http:// dx.doi.org/10.1016/j.ajic.2008.03.002-Medline.

2. Centers for Disease Control and Prevention. CDC/ NHSN Surveillance Definitions for Specific Types of Infections [sede Web]. Atlanta: Centers for Disease Control and Prevention; 2014 [consultado 10 Dic 2015]. Disponible en: https://www.cdc.gov/nhsn/pdfs/ pscmanual/17pscnosinfdef_current.pdf

3. W. R. Jarvis. Benchmarking for prevention: The Centers for Disease Control and Prevention's National Nosocomial Infections Surveillance (NNIS) system experience Infection, 2 (2003), pp. 44-48.

4. EPINE Workgroup. EPINE-EPPS 2015 results: «European PrevalenceSurvey of Healthcare-Associated Infectionsand Antimicrobial Use (EPPS)» [sede Web]. Madrid: Sociedad Española de Medicina Preventiva Salud Pública e Higiene; 2015 [consultado 10 Dic 2015]. Disponible en: http://hws. vhebron.net/epine/Global/EPINE-EPPS\%202015\%20 Informe\%20Global\%20de\%20Espa\%C3\%B1a\%20 Resumen.pdf

5. Ministerio de Sanidad, Servicios Sociales e Igualdad. Guía de Práctica Clínica para la Seguridad del Paciente Quirúrgico [sede Web]. Madrid: Ministerio de Sanidad, Servicios Sociales e Igualdad; 2010 [consultado 10 Dic 2015]. Disponible en: http://www.guiasalud.es/GPC/ GPC_478_Seguridad_Paciente_AIAQS_compl.pdf

6. Ministerio de Sanidad Servicios Sociales e Igualdad. Revisión Sistemática de Eventos Adversos y Costes de la No Seguridad. Las Infecciones asociadas a la Atención Sanitaria [sede Web]. Madrid; 2015 [consultado 10 Dic 2015]. Disponible en: http://www.seguridaddelpaciente. es/resources/documentos/2015/COSTES\%20DE\%20 LA\%20NO\%20SEGURIDAD_Infecciones.pdf

7. J. W. Alexander, J. S. Solomkin, M. J. Edwards. Updated recommendations for control of surgical site infections. Ann Surg., 253 (2011), pp. 1082-1093 http://dx.doi. org/10.1097/SLA.0b013e31821175f8 Medline.

8. D. J. Anderson,K. Podgorny, S. I. Berríos-Torres, D. W. Bratzler, E. P. Delinger, L. Greene Strategies to prevent surgical site infections in acute care hospitals: 2014 Update Infect Control Hosp Epidemiol., 35 (2014), pp. 605627 http://dx.doi.org/10.1086/676022 Medline.

9. J. Ruiz Tovar, J.M. Badia. Prevention of surgical site infection in abdominal surgery. A critical review of the evidence Cir Esp., 92 (2014), pp. 223-231 http://dx.doi. org/10.1016/j.ciresp.2013.08.003 Medline.

10. American Hospital Association. Surgical site infection (ISQ) change package. Preventing surgical site infections [sede Web]. Chicago; 2014 [consultado 10 Dic 2015]. Disponible en: http://www.hret-hiin.org/resources/display/ surgical-site-infections-change-package

11. D. W. Bratzler, D. R. Hunt - The surgical infection prevention and surgical care improvement projects: National initiatives to improve outcomes for patients having surgery. Clin Infect Dis., 43 (2006), pp. 322-330 http:// dx.doi.org/10.1086/505220 Medline.

12. J. E. Keenan, P. J. Speicher, J. K. M. Thacker, M. Walter, M. Kuchibhatla, C. R. Mantyh. The preventive surgical site infection bundle in colorectal surgery: An effective approach to surgical site infection reduction and health care cost savings. JAMA Surg., 149 (2014), pp. 1045-1052 http://dx.doi.org/10.1001/jamasurg.2014.346 Medline.

13. W. Lutfiyya, D. Parsons, J. Breen. A colorectal 'care bundle' to reduce surgical site infections in colorectal surgeries: A single-center experience - Perm J., 16 (2012), pp. 10-16 Medline.

14. Rusell, Maria Lucia; Gallo, Maria Claudia; Hojman, Martin; Torres, Marta "Estudio del Consumo de Antimicrobianos (ATM) en las Unidades de Terapia Intensiva (UTI) y Clínica Médica (CM) del Hospital Rivadavia durante el periodo 2013- 2016 : Diagnóstico de Situación" - XXVIII Jornadas Cientificas 2017 Asociacion De Profesionales Del Hospital Rivadavia.

15. Moylan JA, Kennedy BV. Importance of surgical gowns and drapes as barriers for prevention of postoperative infections - Minerva Chir. 1981 Oct 31;36(20):1365-72.

16. Guía para la prevención de Infecciones relacionadas al sitio quirúrgico y profilaxis antibiótica prequirúrgica- Instituto Nacional de Epidemiología - 2010.

17. Consenso Interinstitucional "Actualización Sobre Medidas De Prevención De Infecciones De Sitio Quirurgico" - Ministerio de Salud Administración Nacional de Laboratorios e Institutos de Salud (ANLIS) Instituto Nacional de Epidemiología (INE) Sociedad Argentina de Infectología (SADI) Guía para la prevención de Infecciones relacionadas al sitio quirúrgico y profilaxis antibiótica prequirúrgica- Instituo Nacional de Epidemiología 2010Agosto 2015.

18. Guideline for Hand Hygiene in Health-Care Settings Recommendations of the Healthcare Infection Control Practices Advisory Committee and the HICPAC/SHEA/ APIC/IDSA Hand Hygiene Task Force.

19. Lipp A, Edwards P. Disposable surgical face masks: a systematic review. Can Oper Room Nurs J. 2005 Sep;23(3):20-1, 24-5, 33-8. 
20. M. W. Skinner, B. A. Sutton. Do Anaesthetists Need to Wear Surgical Masks in the Operating Theatre? A Literature Review with Evidence-Based Recommendations - Anaesth Intensive Care 2001; 29: 331-338.
Impact of an infection prevention program on the

behavior of surgeons and anesthetists in a public hospital

Introduction: Surgical site infections (SSI) occupy a prominent place in nosocomial infection surveillance and control programs. There is evidence that up to $60 \%$ could be avoided. Our objective was to determine if the percentage of compliance with surgical infection prevention measures is higher after the implementation of a program in a public hospital.

Methods: Intersubject, observational, retrospective and crosssectional comparative study. Record of compliance with prevention measures before and after instrumentation of the Program were analyzed. The Program consisted of: delivery of surgical clothing upon admission to the operating room, dissemination of SSI prevention recommendations, daily audit. Variables: professional category, proper use of surgical mask, scrubs, and surgical gowns, surgical hand hygiene, change of clothes when stained, and handling of personal effects within the operating room. The data were analyzed using VCCstat 3.0. $95 \%$ confidence intervals were estimated.

Results: In the control "Professional Category" variable, there were significant differences in all audited variables between surgeons and anesthetists, therefore, a separate population analysis was performed.

Surgeons and anesthetists improved the compliance of both adequate, change of clothes when stained and handling of personal effects within the operating room. The anesthetists also presented significant improvements in proper use of chinstrap and surgical hand hygiene.

Discussion: Program implementation was successful. Continuous efforts in the continuation of improvement is important. The effect on ISQ rates is a fact to be measured.

Key words: Prevention measures, surgical infections, operating room behavior, anesthetists, surgeons. 